\title{
Research on the Simulation Design of Humanistic Landscape Optimization in Urban Residential Area Based on Computer Technology
}

\author{
Wei $\mathrm{Wu}^{1,2}$, Boxun Wang ${ }^{2}$ and Shuai Yang ${ }^{2}$ \\ ${ }^{1}$ He Xiangning College of Art and Design, Zhongkai University of Agriculture and Engineering \\ Guangzhou 519000, Guangdong Province, China \\ https://www.zhku.edu.cn \\ ${ }^{2}$ Faculty of Innovation and Design, City University of Macau, Macau \\ http://www.cityu.edu.mo \\ E-mail: 50958250@qq.com
}

Keywords: computer technology, urban residential area, humanistic landscape, a model of optimization design, future urban landscape design

Received: July 15, 2019

\begin{abstract}
With economic development, individuals are paying increasing attention to their surrounding living setting. They attach excellent significance to urban design coordination and the general natural environment, and follow a lifestyle that is easy and comfortable. In the past, scientists concentrated on the physical shape of residential areas and landscape design, and few quantitative research on ecological housing fields ' color modifications have been conducted. The human landscape optimization design of urban residential areas is performed on the basis of computer technology. The urban landscape design of residential urban landscapes was carried out from various stages. Comparison is made between the threedimensional color and three-dimensional color rates of two distinct model plant color landscape models, and the fundamental requirements for rational landscape color plant distribution are acquired. A computer-based model of artificial landscape layout is suggested for urban residential areas. It offers a theoretical foundation for future design of urban landscape
\end{abstract}

Povzetek: Predstavljena je raziskava urbanega okolja v smislu optimizacije več parametrov.

\section{Introduction}

The residential areas in almost every city will have more or less humanistic landscape. These humanistic landscapes are the organic combination of the humanities and the landscape. Humane landscape is one of the indispensable conditions to improve the quality of urban life. It enables residents to enjoy the natural scenery and the aesthetic pleasure, feel the edification of culture and improve the taste of life in daily life. For example, some greening and water bodies in the city, as well as various facilities, not only have their basic material functions, for example, green landscaping can improve urban greening, absorb carbon dioxide, clean air, and reduce soil erosion, but also has the function of spirit in the shape and design, they are more artistic, which can give people bring aesthetic pleasure enjoy. Through humanistic landscape design, the material and spiritual functions of these landscapes can be maximized. Because the humanities landscape design not only can show the integrity and ecology of the landscape, but also fully display its artistic and comfortable. The design of humanistic landscape in urban residential area accords with the needs of modern people's living environment, improves people's living environment, and is also conducive to the protection of natural environment, so that people can get along well with the environment [1].

\section{Literature review}

IMcharg has ever put forward an important idea of the design of urban residential environment and humanistic landscape, namely the idea of comprehensive ecological planning. In the book "water landscape", Rosemarie Mike Lily focuses on the design of the garden waterscape. In the book "ecological design and comprehensive treatment of urban waterscape" NARS: creating a clear and beautiful original waterscape system, JinyuanHuan focuses on the analysis of waterscape's ecological design concept and how to carry out water ecological design. He pointed out the shortage of modern urban waterscape, analyzed the reasons for the problems, and put forward the "NARS ecological waterscape system". BaiYufang and Chen Wangqing discussed the application of the virtual Waterscape in the application of the waterscape of Hangzhou residential district [2].

In recent years, great achievements have been made in the construction of urban residential areas in China. However, it is also faced with the problems of large residential area, excessive consumption of resources and energy, and low ecological degree of residential areas. Therefore, while China is actively promoting the "lowcarbon economy" growth mode, increasing the proportion of ecotype residential areas is the inevitable direction of the development of residential areas. At present, the 


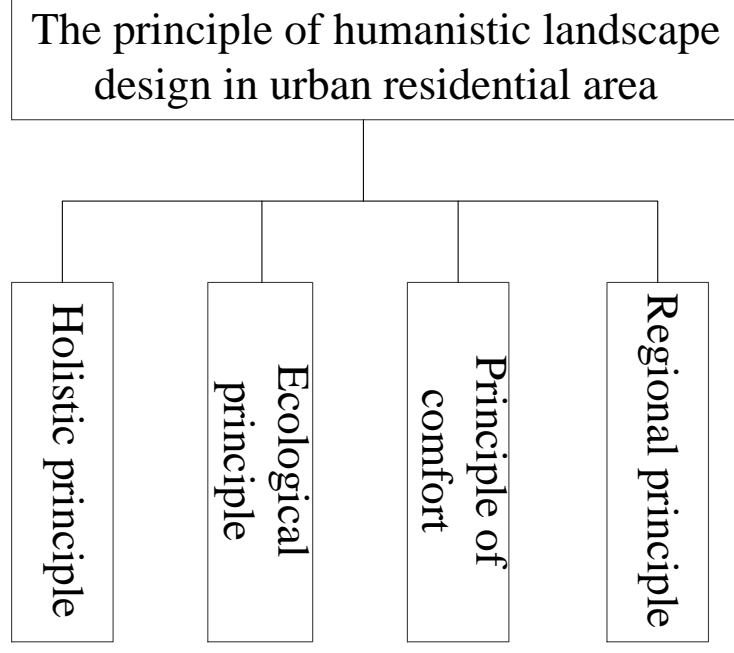

Figure 1: Principle of humanistic landscape design in urban residential area.

research on Eco residential area in China is not deep enough. Under the urgent task of building eco residential area, we need to strengthen the research on the theory of ecological residential area. Figure 1 is the principle of humanistic landscape design in urban residential areas.

\section{Research methodology}

Optimization simulation design of humanistic landscape in urban residential area based on computer technology

\subsection{Design principle}

In the process of three-dimensional image design of humanistic landscape in urban residential area, the urban landscape and landforms are obtained first, and the characteristics of humanistic landscape plant configuration are obtained. The stereoscopic index of plant colorization in urban residential area is set up, and the three-dimensional image design of humanistic landscape in urban residential area is completed based on this. The detailed steps are as follows:

CI represents the environmental factors of the humanistic landscape before the design. Hp represents the ecological factors before the design of city residential landscape. $\mathrm{M}_{\mathrm{F}}$ represents the natural factors. $\vartheta_{v}$ represents human factors. [3] Formula (1) is used to obtain the characteristics of humanistic landscape configuration.

$$
d f^{\prime \prime \prime}=\frac{\left(C I \cdot H_{p}\right)}{\widetilde{\omega} \times \Psi} M_{\Gamma}
$$

In the formula, $\tilde{\omega}_{\text {represents the function of }}$ humanistic landscape in urban residential area, and $\psi$ represents the physiological and ecological characteristics of color plants.

Assuming that $K$ (乡) represents the equal number of color plants, $\mathrm{C}(\mathrm{i}, \mathrm{t})$ represents the physical form of urban road landscape.The formula (2) is used to give the threedimensional index of the color of urban plants.

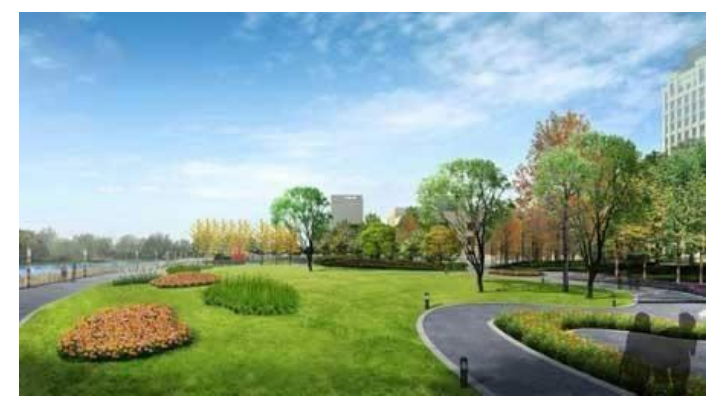

Figure 2: The rational collocation of color plants in the cultural landscape.

$$
K_{p}(\varphi)=\frac{K_{(\xi)} \times C(i, t)}{\sum_{i, j}^{m} \omega(\gamma) \cdot \theta(\mathfrak{I})} \otimes \beta(o p)
$$

In the formula, $\omega(\gamma)$ represents the physical effect of the color of urban landscape plants. $\theta(\mu)$ represents the greening level of the city. $\beta(\mathbf{o p})$ is the characteristics of leaf color leafed plants.

$h_{j}$ represents the color leafed plants leaves. $\tau$ (II) represents the proportion of different colors of leaf plants to the whole. $M_{k}$ represents the comprehensive evaluation of color leafed plants adaptability equation. The formula (3) is used to form a three-dimensional image design model of the urban landscape plant landscape.

$$
P_{\Sigma} \quad(x y)=\frac{\lambda(i y)}{d f^{\prime \prime \prime} \times K_{p}(\varphi)} \times\left[h_{j} \cdot \tau(l l)\right] M_{k}
$$

In the formula, $\lambda(i y)$ represents the variety of color plants and their growth adaptability.

However, traditional methods do not consider the seasonal variation of color and the physiological and ecological characteristics of plants.

\subsection{Three-dimensional image optimization design of humanistic landscape in urban residential area}

(1) Rational collocation of color plants in the cultural landscape

Plants commonly used in urban greening plants contain various kinds of trees, herbs and shrubs. They are the basic elements of urban ecological and cultural environment, as shown in Figure 2. According to the current urban environmental conditions and existing plant resources and their Greening Status, color symbolization, color Psychological Association, the configuration is carried out in the process of establishing the optimization model of the humanistic landscape design. In the configuration of color plants, seasonal variation of plant biology characteristics and color are combined. And according to the constraint conditions of humanistic landscape design in the residential area of urban residents, the landscape elements of the landscaping of the plants are obtained. The detailed steps are as follows:

$\mathrm{v}(\mathrm{ol})$ represents the natural landscape elements of the city before the landscape design. $\varphi_{o}^{k}$ represents the environmental conditions of the city. ku represents

existing plant resources. $\mathbf{c}(\boldsymbol{\nabla})$ represents the main body of the urban green space system. The formula (4) is used 
to obtain the basic conditions for the rational collocation of color plants in the landscape.

$$
q(r, y)=\frac{c(\nabla) \cdot f(X C)}{\varphi_{o}^{k} \times H H_{k}} \bigoplus \frac{k(\omega)}{\varepsilon(o, p)} \iota(e l)
$$

In the formula, $\mathrm{f}(\mathrm{XC})$ represents the physical effect of plant color. $H H_{k}$ represents the symbolization of plant color. $\mathrm{k}(\mathrm{w})$ represents the geographical latitude and terrain of the greening site. $\varepsilon(\mathbf{0}, \mathbf{p})$ represents the coordination among plant populations, and $\tau(e l)$ represents the species of color plants.

It is assumed that $h$ (uy) represents the basic principles of rational collocation of plants. $\mathrm{m}(\mathrm{l})$ represents the seasonal variation rule of color plants. The formula (5) is used to get the elements of landscaping.

$$
F^{l}(C, X)=\frac{m(l) \times h(u y)}{\zeta(\vartheta) \cdot r(l)}
$$

In the formula, $\zeta(\vartheta)$ represents the mutual coordination between the color plant population, and $\gamma(l)$ represents all the features of the landscape.

It is assumed that $f r$ is the form of plant color expression. $k l$ represents the effect of color patches in urban humanistic landscape, and the color and vegetation characteristics of color plants are obtained by using formula 6 .

$$
\theta(x)^{*}=\frac{\xi(i) \times j k^{*}(v) \bar{\omega}_{k}}{f r \times b n(l p) k l} \cdot q(r, y) \otimes \frac{E(k k)}{F^{l}(C, X)}
$$

In the formula, $\xi(i)$ represents the urban green space system. $j k$ represents the color leafed plants adaptability. $\omega_{k}$ is city lottery leaf plant resources. $v$ represents the principle of urban characteristics. bn(lp) represents plant planting requirements.

The above analysis can show that in the process of establishing the optimization model of the urban humanistic landscape plant color design, the basic conditions for reasonable collocation of plants in the landscape are obtained by the principle of highlighting the urban characteristics and ecology. According to the needs of the landscape theme, the colorful landscape elements of the landscape plants are obtained, and the seasonal variation of color plants is obtained. The physiological and ecological characteristics of the color plants are given, which lays the foundation for the optimization design of the three-dimensional image of the urban landscape plant landscape [4].

(2) Optimization design of three-dimensional image of humanistic landscape in urban residential area

Based on the physiological and ecological characteristics of color plants obtained above, the threedimensional color quantity concept is proposed based on the three-dimensional image optimization design of plant landscape in urban cultural landscape, and the threedimensional color amount of urban humanistic landscape is calculated. The three-dimensional color quantity is used to optimize the three-dimensional image of the urban landscape. The detailed steps are as follows:

It is assumed that $\gamma(\mathbf{n n})$ represents the color leafed plants. $M(n k)$ represents the characteristics of landscape design as a whole and contour. Based on the physiological and ecological characteristics of color plants obtained above, we use the formula (7) to describe the three dimensional spatial structure index of urban humanistic landscape.

$$
(n k)_{k k}{ }^{(M)}=\frac{\sum(\kappa j) \otimes \zeta(\mathrm{km})}{\gamma(n n) \oplus M(n k)} \times F^{l}(C, X)
$$

In the formula, $(\mathrm{kJ})$ represents the three dimensional Green amount, and the $\zeta(\mathrm{km})$ represents the crown diameter.

It is assumed that $\varphi(\mathbf{j} \mathbf{l})$ represents the non threedimensional color leafed plants. $\Psi(j l)$ represents threedimensional color leafed plants. The formula (8) is used to calculate the amount of color leafed plants:

$$
C G(H)=\frac{\varphi(j l) * \psi(j l)}{(n k)_{k k}(M)} \times R(k, l) \otimes v(i, l)
$$

In the formula, $\mathrm{R}(\mathrm{k}, \mathrm{l})$ represents the color of the actual plant, $v(i, l)$ represents the classification of color leafed plants by leaf color characteristics of the situation.

It is assumed that ${ }^{l} \tau$ stands for the three dimensional color sum of common leaf, bicolor and spotted leaves. $\mathrm{m}(\mathrm{k}, \mathrm{l})$ represents the crown of color plants, and $\delta(\mathbf{k}, \mathbf{0})$ represents the three dimensional color sum of autumn leaf, new leaf color, common leaf, bicolor, and leaf color. The $\mathrm{m}(\mathrm{k}, \mathrm{l})$ is defined as the amount of color leafed plants in spring, and the $\delta(\mathbf{k}, \mathbf{0})$ is defined as the color leafed plants in summer and winter. The formula (9) was used to calculate the three-dimensional color conversion rate of urban landscape plants.

$$
\varphi_{l}{ }^{o}(n, j)=\frac{m(k, l) \cdot l_{\tau}}{\delta(k, o)} C G(H) \cdot \frac{n f(b)}{\xi(v v)}
$$

In the formula, $\mathrm{nf}(\mathrm{b})$ represents all the colorful plants of the urban humanistic landscape, and $\xi(v v)$ represents the sum of the three-dimensional color of all the colorful plants of the city's humanistic landscape [5].

It is assumed that $1(b, m)$ represents the sum of the three dimensional Green quantities of non-colored plants. $\lambda(m, n)$ represents the difference in the contrast of three dimensional coloring rate in spring. The model of the three-dimensional image optimization of the urban landscape plant landscape was established by using the type (10).

$$
A(C B)=\frac{\lambda(m, n)}{l(b, m)} \times \varphi_{l}^{o}(n, j) M^{*}
$$

In the formula, $\mathbf{M}^{*}$ represents the number of different phase accumulation value of colorful plants. 


\section{Simulation results and analysis}

In order to prove the validity of the proposed 3D image optimization design model of urban humanities landscape, an experiment is needed. In the environment of Matlab, a simulation platform for three-dimensional image optimization of urban humanities landscape is built. The data of humanistic landscape resources in urban residential areas from May 2016 to October 2017 are the experimental data [6].

\subsection{Different models of landscape design color and three-dimensional color comparison}

This paper uses the model proposed in this paper and the literature model to carry out the experiment of the landscape design of urban landscape plants. The threedimensional color and three-dimensional color rate of plant color landscape design were compared between the 2 different models. The comparison results are shown in figures 2 and 3.

From the analysis of figures 3 and 4, it can be concluded that the color and rate of color rendering of urban humanistic landscape plants is better than that of the literature model. This is mainly because that when we use this model to design the 3D image of plant landscape in urban landscape, we first get the basic conditions of rational collocation of plants in landscape based on the principle of highlighting urban characteristics and ecology. According to the needs of the landscape theme, the colorful landscape elements of the landscape plants are obtained, and the seasonal variation of color plants is obtained. The physiological and ecological characteristics of the color plants are given, which ensures the color rate and three-dimensional color of the three-dimensional image design of the urban landscape plant landscape [7].

\subsection{Comparison of the effectiveness of different models for landscape design}

The model in this paper and literature model were used to design the experiment respectively. The stability (\%) and efficiency $(\%)$ of the 2 different models for the rational allocation of urban cultural landscape were compared [8]. The comparison results were used to measure the overall effectiveness of the 2 different models of urban humanistic landscape design. The results were shown as figure 5 and figure 6.

From the analysis of Figure 5 and figure 6, it can be concluded that the overall superiority of the urban humanistic landscape design using this model is better than the model in the literature. In this paper, the threedimensional color quantity concept of the urban humanistic landscape is designed, and the threedimensional color quantity of the urban humanistic landscape is calculated. The three-dimensional image of urban humanistic landscape is optimized by threedimensional color, which ensures the overall superiority of the landscape design.

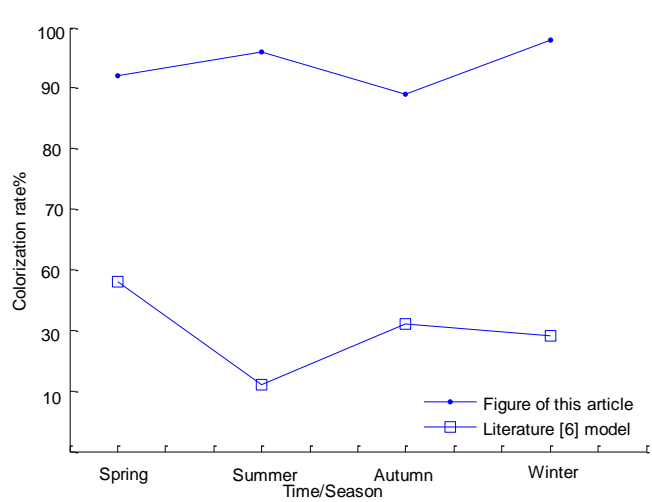

Figure 3: Color comparison of different models of landscape design.

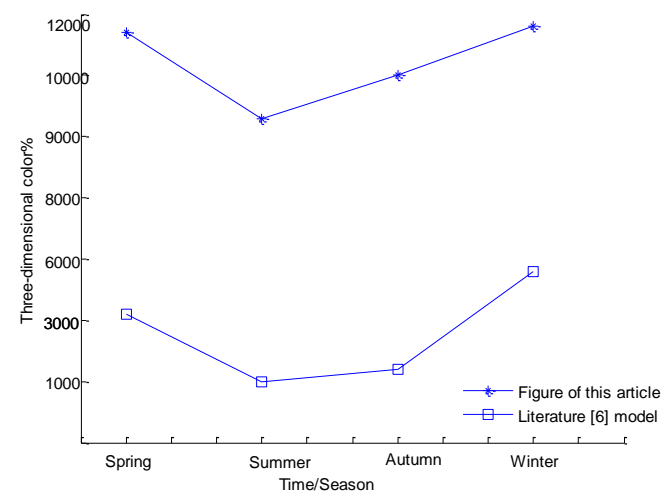

Figure 4: Three dimensional color comparison of different models of landscape design.

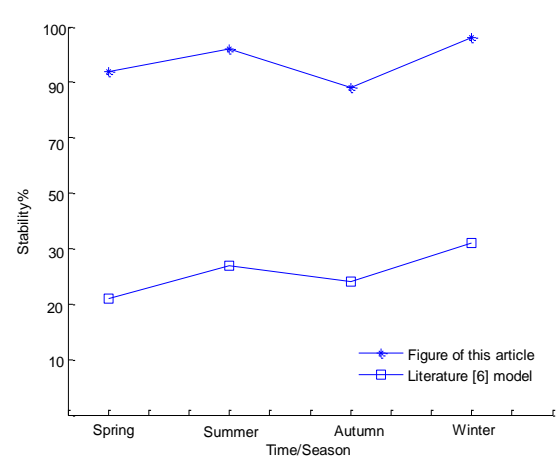

Figure 5: Comparison of the stability of different models of landscape design.

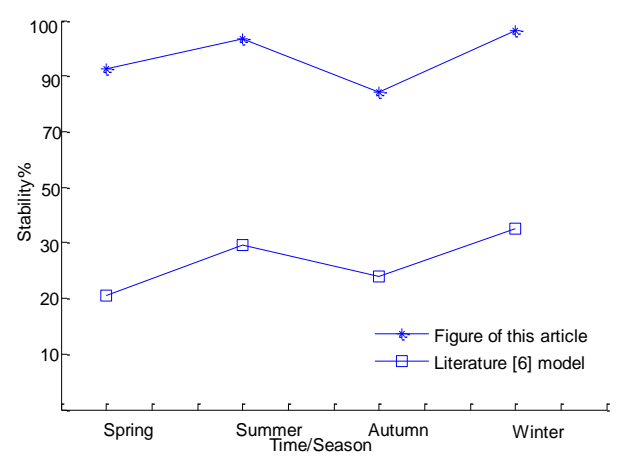

Figure 6: Comparison of the efficiency of different models of landscape design. 


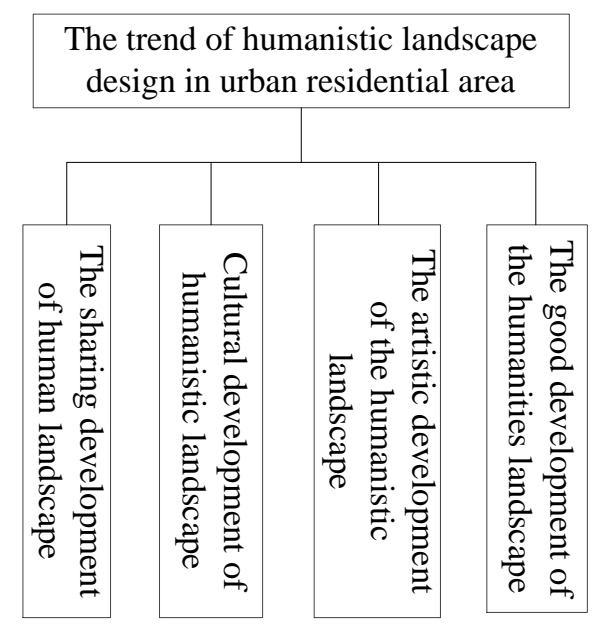

Figure 7: The trend of humanistic landscape design in urban residential areas.

\section{The trend of humanistic landscape design in urban residential areas}

With the accelerated process of urbanization in China, the design of humanistic landscape in urban residential areas is also developing. City residential area cultural landscape is more geared towards life, which meets the needs of the residents in the direction of development. It tries to improve the quality of the entire urban residential area with the most shared, cultural and artistic humanistic landscape. As shown in Figure 7.

(1) The development of the shared cultural landscape

When designing the residential landscape, we should take into account the needs of all users, and design a shared cultural landscape, so that every resident can enjoy and enjoy these cultural landscapes together.

(2) Cultural development of the humanistic landscape

In the design of the humanistic landscape, it is necessary to integrate more history and culture on the basis of the natural environment, so as to make the cultural landscape richer. Natural landscapes are naturally formed, while humanistic landscapes are designed by human beings, and human history and cultural development are the source and foundation of design. Therefore, humanistic landscape is actually a human cultural landscape. Because of the unique cultural background and historical background, the residents can feel the edification of art and historical culture when they appreciate the cultural landscape. Therefore, in the process of development, the pursuit of culture is an important direction in the development of humanistic landscape.

(3) The artistic development of the humanistic landscape

(4) The good development of the humanities landscape

In order to attract household occupancy to a greater extent, many residential areas introduce the water system to the construction of humanistic landscape. It can realize the scientific and rational arrangement of each layout, which can realize the harmonious development of human, environment and society.
With the development of social economy, people's pursuit of art is higher. Therefore, in the process of design, humanistic landscape needs to satisfy people's artistic pursuit and aesthetic need. The humanistic landscape not only maintains the natural ecology, but also has the artistic beauty [9].

\section{Conclusion}

With the continuous improvement of people's living standards, the demand for the living environment is becoming higher and higher. Therefore, the design of the green landscape in the residential area can not only meet the needs of the residents for the green space, but also improve the beauty and landscape culture of the residential area. More importantly, it can play a very important role in the greening of the city and the improvement of the ecological environment. We should pay attention to the ancient and emerging disciplines of the cultural landscape. This design accords with the modern and practical function of the poetic urban garden new space, and creates the beautiful landscape human settlement ecological environment. This should be the goal of all planners.

\section{References}

[1] Jaeger J A G, Bertiller R, Schwick C, et al. Urban permeation of landscapes and sprawl per capita: New measures of urban sprawl[J]. Ecological Indicators, 2010, 10(2):427-441.

https://doi.org/10.1016/j.ecolind.2009.07.010

[2] Smallbone L T, Luck G W, Wassens S. Anuran species in urban landscapes: Relationships with biophysical, built environment and socio-economic factors[J]. Landscape \& Urban Planning, 2011, 101(1):43-51. https://doi.org/10.1016/j.landurbplan.2011.01.002

[3] Mckinney R A, Raposa K B, Cournoyer R M. Wetlands as habitat in urbanizing landscapes: Patterns of bird abundance and occupancy $[\mathrm{J}]$. Landscape \& Urban Planning, 2011, 100(1-2):144152.

https://doi.org/10.1016/j.landurbplan.2010.11.015

[4] Mcdonald R I, Forman R T T, Kareiva P, et al. Urban effects, distance, and protected areas in an urbanizing world[J]. Landscape \& Urban Planning, 2009, 93(1):63-75.

[5] https://doi.org/10.1016/j.landurbplan.2009.06.002

[6] Peterson M N, Thurmond B, Mchale M, et al. Predicting native plant landscaping preferences in urban areas[J]. Sustainable Cities \& Society, 2012, 5(1):70-76. https://doi.org/10.1016/j.scs.2012.05.007

[7] Devitt D, Morris R, Gianquinto G P, et al. Sustainable water use in urban landscapes in the $21 \mathrm{st}$ century: a Las Vegas perspective.[J]. ActaHorticulturae, 2010, 881(881):483-486. https://doi.org/10.17660/ActaHortic.2010.881.77

[8] TarekRashed, JohnWeeks. Assessing vulnerability to earthquake hazards through spatial multicriteria 
analysis of urban areas[J]. International Journal of Geographical Information Systems, 2003, 17(6):547-576.

https://doi.org/10.1080/1365881031000114071

[9] Kilpatrick H J. Effects of archery hunting on movement and activity of female white-tailed deer in an urban landscape. WildlSocBull[J]. Wildlife Society Bulletin, 1999, 27(2):433-440.

[10] Endterwada J, Kurtzman J, Keenan S P, et al. Situational Waste in Landscape Watering: Residential and Business Water Use in an Urban Utah Community[M]. American Water Resources Association, 2008.

[11] https://doi.org/10.1111/j.1752-1688.2008.00190.x 